\title{
Inflammation and Overlap of Irritable Bowel Syndrome and Functional Dyspepsia
}

\author{
Congzhen Wang and Xiucai Fang* \\ Department of Gastroenterology, Peking Union Medical College Hospital, Chinese Academy of Medical Sciences and Peking Union Medical \\ College, Beijing, China
}

Irritable bowel syndrome (IBS) and functional dyspepsia (FD) are common functional gastrointestinal disorders (FGIDs) and account for a large proportion of consulting patients. These 2 disorders overlap with each other frequently. The pathogenesis of IBS or FD is complicated and multi-factors related, in which infectious or non-infectious inflammation and local or systemic immune response play significant roles. There are few studies focusing on the mechanism of inflammation in patients with overlap syndrome of irritable bowel syndrome and functional dyspepsia (IBS-FD). This review focuses on current advances about the role of inflammation in the pathogenesis of IBS and FD and the possible mechanism of inflammation in IBS-FD.

(J Neurogastroenterol Motil 2021;27:153-164)

\section{Key Words}

Dyspepsia; Inflammation; Irritable bowel syndrome

\section{Introduction}

As common functional gastrointestinal disorders (FGIDs), irritable bowel syndrome (IBS) and functional dyspepsia (FD) are lack of gastrointestinal organic lesions, but seriously affect the quality of life of patients. According to the epidemiological data, 26.7-48.7\% of IBS patients and $20.0-42.1 \%$ of FD patients have overlapping symptoms. ${ }^{1-3}$ Overlap syndrome leads to more serious clinical manifestations, worse quality of life and more difficult therapy. ${ }^{4}$ The symptoms of IBS or FD are usually induced by diet, gastrointestinal infection, gut microbiota alteration, stress, psychological disorders and other unknown factors. The chronic inflammation after infection or non-infectious inflammation related to the above factors and immune response lead to visceral hypersensitivity, dysfunction of brain-gut axis and intestinal mucosal barrier, which may be the causes of IBS or FD symptoms. However, whether inflammation has the same mechanism in overlap syndrome of IBS and FD (IBS-FD) remains unclear and there are few related studies to confirm this. In this article, we reviewed recent advances about mechanism of inflammation in IBS-FD and provided references about the possible mechanism of inflammation in IBS-FD.

\section{The Role of Gastrointestinal Infection in the Pathogenesis of Irritable Bowel Syn- drome, Functional Dyspepsia, and Overlap Syndrome of Irritable Bowel Syndrome and Functional Dyspepsia}

In the population suffering acute gastrointestinal infection, the

Received: August 7, 2020 Revised: November 23, 2020 Accepted: November 29, 2020

() This is an Open Access article distributed under the terms of the Creative Commons Attribution Non-Commercial License (http://creativecommons. org/licenses/by-nc/4.0) which permits unrestricted non-commercial use, distribution, and reproduction in any medium, provided the original work is properly cited.

*Correspondence: Xiucai Fang, MD Department of Gastroenterology, Peking Union Medical College Hospital, Chinese Academy of Medical Sciences and Peking Union Medical College, 1 \# Shuaifuyuan, Wangfujing, Dongcheng District, Beijing 100730, China

Tel: +86-10-6915-6892, +86-1343-913-6086, Fax: +86-10-6915-1963, E-mail: fangxiucai2@aliyun.com 
prevalence of post-infectious IBS (PI-IBS) and post-infectious FD (PI-FD) is $12.7 \%$ and $9.5 \%$, while the odds ratio of IBS and FD following gastrointestinal infection is 3.5 and 2.5 , respectively. ${ }^{5}$ According to previous studies, pathogens including Shigella, Salmonella, Escherichia coli, Campylobacter jejuni, Vibrio cholerae, Clostridium difficile, norovirus, Giardia lamblia, and Trichinella spiralis were considered to be associated with IBS, while pathogens that correlated with FD included Helicobacter pylori, Shigella, Salmonella, E. coli, C. jejuni, C. difficile, norovirus, and G. lamblia.-7 A recent prospective cohort study found that the incidence of PIIBS, PI-FD, and IBS-FD was $16.5 \%, 7.4 \%$, and $4.7 \%$, respectively, 1 year after acute gastrointestinal infection, and the difference was statistically significant compared with healthy controls without infection history. ${ }^{8}$ Mearin et al found that $36.0 \%$ of all the patients with PI-FD or PI-IBS 1 year after the outbreak of acute Salmonella gastroenteritis had overlapping symptoms. The incidence of IBS-FD 3 years after acute $G$. lamblia infection was higher than the control group without infection (44.0\% vs $29.0 \%)$. ${ }^{10}$ Spiller $^{11}$ put forward the hypothesis that the site of acute infection may be related to the outcome of symptoms in post-infectious FGIDs. If the infection is limited to the proximal intestine, patients are more likely to develop symptoms of FD; if the distal intestine or colon is involved, symptoms of IBS may occur. When the proximal and distal intestine are both involved, patients are more likely to develop overlap syndrome of IBS and FD. ${ }^{11}$ However, there is a lack of prospective studies to confirm the above hypothesis.

In the months to years after acute gastrointestinal infection, mild chronic inflammation remained in the gastrointestinal tract and mainly reflected by increase and activation of inflammatory cells such as mast cells (MCs), eosinophils, and macrophages in the mucosa, which was difficult to be found by routine blood tests and endoscopy. ${ }^{12-16}$ It has been proved that the local and systemic immune response concomitant or secondary to inflammation can lead to damage of the intestinal mucosal barrier, dysfunction of enteric nervous system and brain-gut axis, as well as abnormal sensory and motor functions of the gastrointestinal tract. These changes are related to epigastric pain syndrome (EPS) and postprandial distress syndrome of FD in the upper gastrointestinal tract, abdominal pain, and altered bowel habits of IBS in the lower gastrointestinal tract. We will detail evidence of literatures in the following paragraphs.

\section{The Chronic Inflammation and Immune Response Secondary to Gastrointestinal Infection}

After the pathogen of acute infection is removed, although acute mucosal injury is repaired, the ability of the immune system to terminate inflammation is impaired so that mild chronic inflammation is left in the gastrointestinal mucosa of PI-IBS or PI-FD patients. The persistent immune response to mild inflammation of the gastrointestinal mucosa may be involve in the pathogenesis of PI-IBS or PI-FD. A previous study has found that the number of MCs in the terminal ileum mucosa in patients with PI-IBS was significantly increased compared with that in control subjects. ${ }^{12}$ The intraepithelial $\mathrm{T}$ lymphocyte counts in rectum of patients with PI-IBS were still in a high level 1 year after Campylobacter enteritis. ${ }^{13}$ The increase of MCs located within $5 \mu \mathrm{m}$ of intestinal nerve fibers was significantly correlated with severity and frequency of abdominal pain/discomfort in patients with IBS. ${ }^{17}$ Compared with nonspecific FD patients or healthy controls, the histological score of chronic gastric inflammation and the number of activated MCs within $5 \mu \mathrm{m}$ of nerve fibers in the gastric antrum were significantly greater in patients with PI-FD. ${ }^{14}$ It has been confirmed that there existed persisting focal CD8 + T lymphocyte and eosinophil aggregates, decreased CD4 + T lymphocytes and increased macrophage counts surrounding the crypts in the duodenum of patients with PI-FD. ${ }^{15,16}$ Duodenal eosinophilia was proved to be associated with early satiety, ${ }^{18}$ and there was a significant correlation between epigastric burning and the degree of duodenitis in patients with PI$\mathrm{FD} .{ }^{16}$ Inflammatory cells release pro-inflammatory cytokines such as TNF- $\alpha$, IFN- $\gamma$, IL-6, IL-8, IL-18, and anti-inflammatory factors such as IL-10 and IL-13, which are important markers of immune response and play important roles in the regulation of inflammatory cascade. The expression levels of IL-6, IL-18, and IFN- $\gamma$ in colonic and rectal mucosa of patients with PI-IBS were higher than those in controls and patients with non-PI-IBS while the expression level of IL-10 was lower. ${ }^{19,20}$ Compared with the control group, plasma IL-6, IL-18, and TNF- $\alpha$ levels in PI-IBS were significantly higher while the plasma IL-10 level was lower. ${ }^{21,22}$ There is no comparative study about the levels of multiple inflammatory factors between PI-FD and non-PI-FD or healthy subjects.

\section{Damage of Intestinal Mucosal Barrier Associated With Gastrointestinal Infection}

The intestinal mucosal barrier consists of symbiotic bacteria and mucus layer, epithelial cells and cell-cell junctions, as well as the lamina propria containing connective tissue. ${ }^{23}$ In patients with post-infectious FGIDs, pathogens are usually eliminated and the damage of intestinal mucosal barrier is mainly caused by the postinfectious intestinal flora dysbiosis and chronic inflammation. Tolllike receptor (TLR) is a type of receptor protein which is mainly involved in recognizing microbial products, mediating infection- 
related immune response and inflammatory signal transduction. Homologous ligands such as some bacterial components such as flagellin and lipopolysaccharide bind to TLR and mediate the activation of mature MCs and release of inflammatory mediators such as histamine, tryptase and prostaglandin E2, which may be the main mechanism of intestinal flora dysbiosis causing low-grade inflammation. ${ }^{24}$

Flora dysbiosis affect the normal amino acid metabolism, leading to composition changes of the mucus layer and the damage of the mucus barrier. Most of threonine in diet is used to synthesize secretory mucin, and restriction of threonine from diet can reduce the synthesis of secretory mucin in intestinal epithelial cells. ${ }^{25}$ The metabolism of glycine, serine, and threonine is closely related to the abundance of some species of Bacteroides (eg, Bacteroides thetaiotaomicron) and Firmicutes (eg, Faecalibacterium prausnitzii). ${ }^{26,27}$ The abundance of Faecalibacterium including F. prausnitzii in patients with IBS was significantly lower than that of healthy controls. ${ }^{28}$ The microbial diversity of colonic mucosa and feces in PIIBS patients was reduced, ${ }^{29}$ and the Index of Microbial Dysbiosis was correlated with the degree of abdominal pain and increased bowel movements. ${ }^{27}$ In patients with $\mathrm{FD}$, the abundance of anaerobic bacterium including Prevotella, Veillonella, and Actinomyces in duodenal mucosa was lower than that of healthy controls, ${ }^{30}$ but a lack of study was found in the exploration of the abundance change of Bacteroides and Firmicutes in patients with FD.

Tight junctions and adherens junctions are important components of the intestinal mucosal barrier. Tight junctions are protein complexes composed of transmembrane proteins including claudins and occludin, junction adhesion molecules, and intracellular protein zonula occludens (ZO). The intracellular domains of protein complexes are anchored on the cytoskeleton by $\mathrm{ZO}^{31,32}$ Adherens junctions are mainly composed of E-cadherin, catenin and actin. It has been confirmed that the activation of MCs in jejunum mucosa of patients with IBS was correlated with the decreased expression of ZO-1 as well as the degree of diarrhea symptoms. ${ }^{33}$ Activated $\mathrm{MCs}$ release tryptase, which binds to the protease activated receptor (PAR) on the basolateral side of intestinal epithelial cells. The combination of PAR with PAR1 leads to the increase of intestinal epithelial cell permeability through mechanisms such as apoptosis and the activation of myosin light chain kinase (MLCK) resulting in the redistribution of tight junction proteins. ${ }^{34}$ The pro-inflammatory cytokines such as TNF- $\alpha$ released by inflammatory cells can induce the contraction of actin-myosin ring at the top of intestinal epithelial cells, the redistribution of ZO-1 and occludin, the decrease of transepithelial electrical resistance, and the increase of intestinal epithelial cell permeability to macromolecules by inducing the phosphorylation of MLCK. ${ }^{35}$ In patients with FD with infiltration of eosinophils and MCs, the transepithelial electrical resistance of duodenal mucosa decreased, suggesting that the integrity of mucosal barrier was damaged. ${ }^{36}$ Further study found that the expression of ZO-1 and occludin was abnormal and the phosphorylation of serine/threonine residues of occludin reduced, which may be the cause of duodenal mucosal integrity damage. ${ }^{36}$

\section{The Influence of Gastrointestinal Infection on Enteric Nervous System}

There are a large number of MCs adjacent to neuron-specific enolase, substance $\mathrm{P}(\mathrm{SP})$, and 5-hydroxytryptamine (5-HT) positive nerve fibers in the digestive tract mucosa. ${ }^{37}$ The contact between cell membrane of MCs and the axons of adjacent nerve fibers lays a structural foundation for the neuroimmune interactions of the intestinal mucosa. Compared with healthy controls, the number of MCs around the nerve fibers of colon and terminal ileum in patients with PI-IBS increased significantly. ${ }^{12}$ Animal experiments and studies on patients with IBS-D suggested that nerve growth factor (NGF) released by MCs bound with tyrosine kinase A receptor on sensory nerve endings, which promoted the proliferation of nerve fibers expressing transient receptor potential vanilloid type-1 (TRPV1), calcitonin gene related peptide (CGRP), and SP, and improved the activation of TRPV1 in the meantime. Under these circumstances, sensory nerve fibers released more pain-related neuropeptides (such as CGRP and SP) when stimulated, leading to increased visceral sensitivity. ${ }^{38,39}$ Activated MCs also released adenosine triphosphate, prostaglandin, and other inflammatory mediators, which bind to $\mathrm{P} 2 \mathrm{X}$ receptor of purinergic neurons, prostaglandin receptor, and TRPV1 to excite cholinergic motor neurons and promote intestinal movement. ${ }^{40}$

Inflammatory cells such as MCs and eosinophils produce and release $\mathrm{NGF}$ and neurotrophin resulting in local tissue hyperinnervation (neural sprouting, and neural and ganglionic hypertrophy), and a switch in the neurochemical code toward preferential expression of neuropeptides (eg, SP and CGRP) which are frequently present in nociceptive neurons. ${ }^{41,42}$ These changes may be related to visceral hypersensitivity of patients with FD. In addition, the aggregation and degranulation of duodenal eosinophils in patients with FD were correlated with the increase of the density and sprouting of fine nerve fibers in duodenal mucosa, which was more significant in patients with $\mathrm{EPS}^{43}$ Another study pointed out that the infiltration of eosinophils in duodenal mucosa of patients with FD was correlated with the structural change (abnormal ganglion structure 
and gliosis) and functional impairment (decreased calcium responses to depolarization and electrical stimulation) of the submucosal nerve plexus, ${ }^{44}$ which may affect neuronal and muscular functions and lead to clinical symptoms in patients with FD.

Enterochromaffin cells (ECs) concentrate around the afferent nerve endings of gastrointestinal mucosa and synthesize and release 5-HT to the lamina propria. 5-HT regulates gastrointestinal motility through binding to $5-\mathrm{HT}_{2}$ receptor and $5-\mathrm{HT}_{4}$ receptor, meanwhile influencing visceral sensation through binding to the $5-\mathrm{HT}_{3}$ receptor. $^{45,46}$ Increased ECs was one of the acute changes following Campylobacter enteritis which could persist for more than a year ${ }^{47}$ and was proved to be an important independent predictor of developing PI-IBS. ${ }^{48}$ The number of ECs as well as 5 -HT released by them in gastric mucosa of patients with PI-FD were significantly higher than those of patients with non-PI-FD and healthy controls, correlating with the degree of mucosal inflammation. ${ }^{14}$ TNF- $\alpha$ and IFN- $\gamma$ in inflammatory response downregulated the expression of selective 5-HT reuptake transporter, resulting in the decrease of 5-HT reuptake and sustained effect of 5-HT. ${ }^{49}$

\section{The Influence of Gastrointestinal Infection on Brain- gut Axis}

Enteric nervous system interacts with the central nervous system manifesting as emotional and physiological stress that can affect mucosal function of secretion and barrier, increase visceral sensitivity, and change gastric emptying and intestinal transit. Conversely, change of gastrointestinal motility, visceral inflammation and injury amplify the signals of the ascending visceral afferent pathway and affect brain activity, causing more intense pain and emotional/mental disorders including anxiety and depression. ${ }^{50,51}$ There are 2 main neural regulatory pathways involved in visceral sensation. One is the excitatory pain-regulatory pathway, which consists of sensory nerves of the brain (anterior cingulate cortex, insula, hippocampus, amygdala, etc) and spinal cord. The function of this pathway is in facilitation (sensitization) in the visceral hypersensitivity state. ${ }^{52}$ The other is the inhibitory pain-regulatory pathway, which mainly includes the vagal afferent pathway and is in a low state while visceral hypersensitivity occurs. ${ }^{53}$ During a chronic inflammatory period, increased MCs release nociceptive molecules such as protease, histamine, platelet activating factor, leukotriene, cytokines (eg, TNF- $\alpha$, IFN- $\gamma$, IL-1 $\beta$, and IL-6). These inflammatory mediators acted on the adjacent nociceptive dorsal root ganglion neurons to influence the excitability and sensory threshold of neurons and cause visceral hypersensitivity through enhancing the calcium influx of neurons and increasing the firing rates of submucosal neurons. ${ }^{54,55} 5-\mathrm{HT}$, released by ECs, is a type of important mucosal signaling molecule, while those secreted by enteric neurons is an important neurotransmitter, and constitutes significant effects on functions of brain-gut axis. ${ }^{56,57}$ Chronic high concentration of 5-HT binding to the $5-\mathrm{HT}_{3}$ receptors on the nociceptive neurons of the vagus in the colorectal mucosa could enhance the pain perception induced by colorectal distention, which could be blocked by vagotomy or $5-\mathrm{HT}_{3}$ receptor antagonists. $^{46}$

Corticotropin releasing factor (CRF) is mainly produced by the paraventricular nucleus of the hypothalamus, cerebral cortex, hippocampus, amygdala, and locus coeruleus. Its mRNA is highly expressed in the adrenal gland, gastrointestinal tract, thymus, skin, placenta, and inflammatory cells. CRF plays a significant role in the regulation of brain-gut axis. Animal experiments showed that $\mathrm{CRF}$ inhibited gastric contraction and gastric emptying through binding to $\mathrm{CRF}_{2}$ receptors and stimulated colon transit and defecation by activating $\mathrm{CRF}_{1}$ receptors. ${ }^{58}$ Cytokines such as IL-1 $\beta$ and bacterial endotoxins induced by gastrointestinal infection could cross the blood-brain barrier, affect CRF neurons in the hypothalamus and activate the hypothalamic-pituitary-adrenal (HPA) axis. ${ }^{59}$ Overman et $\mathrm{al}^{60}$ found that exposure of pig ileum to specific concentrations of CRF increased the permeability of intestinal epithelial cells by promoting the release of TNF- $\alpha$ and protease from MCs. As a type of neuromodulator, TNF- $\alpha$ excites the neurons of nucleus tractus solitarii and inhibits gastric motility through the vago-vagal reflex pathway, which may be related to delayed gastric emptying in FD or overlap syndrome. Hussain et $\mathrm{al}^{61}$ established the animal model of IBS-D overlapping with FD by intraperitoneal injection of CRF. They also found that trimebutine significantly regulated gastrointestinal motility by promoting gastric emptying as well as reducing bowel movements. ${ }^{62}$ It is reasonable to speculate the mechanism of trimebutine may be relevant to inhibition of CRF.

Taken together, we summarize the possible inflammatory and immune activation in gastrointestinal mucosa of patients with postinfectious IBS-FD in Figure.

\section{Possible Mechanism of Helicobacter pylori Infection in Overlap Syndrome of Irritable Bowel Syndrome and Functional Dyspepsia}

Although meta-analysis does not support a specific association between IBS and $H$. pylori infection, ${ }^{63}$ some evidences suggest that $H$. pylori infection may be involved in the pathogenesis of IBS. The positive rate of $H$. pylori, cytotoxin-associated gene A, and vacuolating cytotoxin A alleles (eg, s1 and s2) in patients with IBS$\mathrm{D}$ were significantly higher than those in healthy controls. ${ }^{64}$ Both 


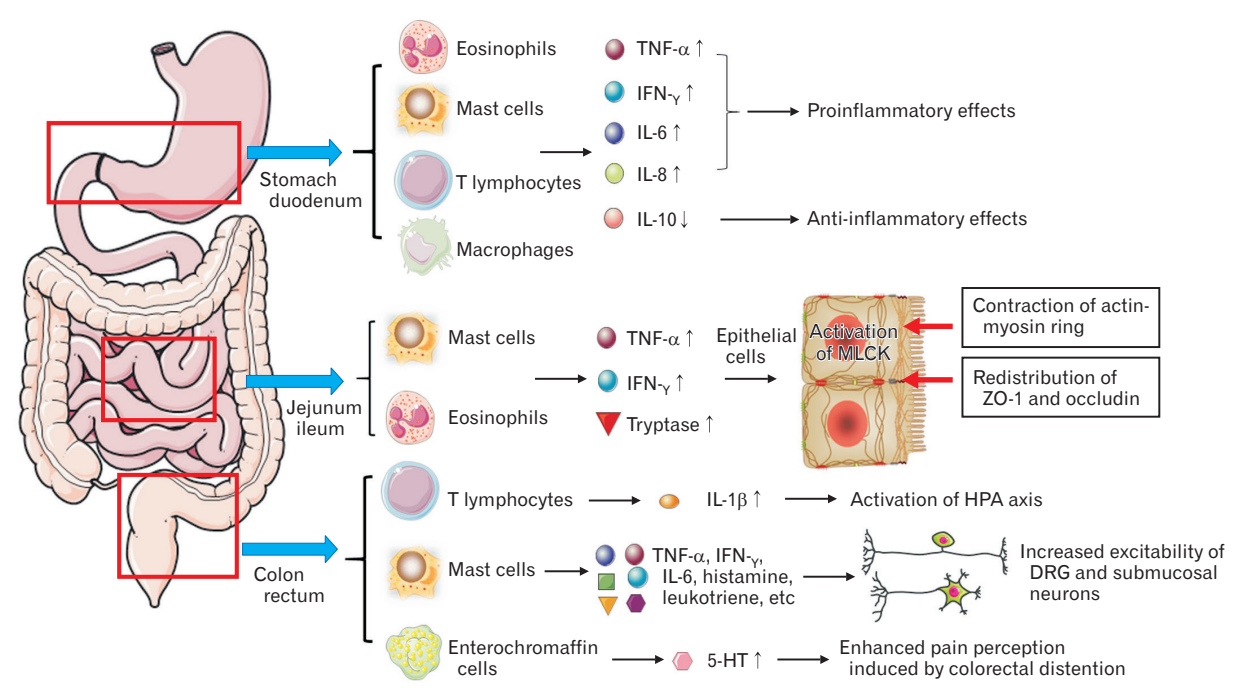

Figure. The possible inflammatory and immune activation in gastrointestinal mucosa of patients with post-infectious irritable bowel syndromefunctional dyspepsia. Inflammatory cells (mast cells, eosinophils, T lymphocytes, and chromaffin cells) are activated and release inflammatory factors (TNF- $\alpha$, IFN- $\gamma$, IL-1/6/8, etc) and inflammatory mediators (histamine, leukotriene, platelet activating factor, 5-hydroxytryptamine [5$\mathrm{HT}]$, etc) to participate in the inflammatory response, resulting in the increase of epithelial permeability through the redistribution of tight junction proteins and visceral hypersensitivity through 5-HT and increased excitability of afferent neurons. MLCK, myosin light chain kinase; HPA, hypothalamic-pituitary-adrenal; ZO-1, zonula occludens-1; DRG, dorsal root ganglion.

vacuolating cytotoxin A and neutrophil-activating protein significantly activated MCs and increased the release of pro-inflammatory cytokines such as IL-6. ${ }^{65,66}$ A cross-sectional, observational study has concluded that duodenal lymphocytosis was significantly associated with bloating of FD patients, and the simultaneous presence of duodenal lymphocytosis and $H$. pylori infection was significantly more prevalent in FD patients than in control subjects. ${ }^{67}$ In multivariate analysis, the odds of experiencing severe symptoms in patients with severe microscopic duodenitis was 2.2 times greater than in individuals with very mild, mild, or moderate duodenitis. ${ }^{68}$ A large population study showed that $H$. pylori infection and any dyspepsia-related consultation significantly increased the likelihood of an IBS related consultation. ${ }^{69}$ Duodenal inflammation caused by $H$. pylori infection may play a role in the pathogenesis of patients with IBS-FD, which needs to be confirmed in the future with more studies in patients with IBS-FD.

Mönnikes et $\mathrm{al}^{70}$ showed that discomfort and pain thresholds on gastric distension were lower in $H$. pylori-positive FD patients. These patients also showed higher antral mucosal levels of CGRP and SP, which negatively paralleled the levels of discomfort and pain thresholds, demonstrating the involvement of SP and CGRP in the sensitization of afferent neuronal pathways. ${ }^{70}$ Choi et al ${ }^{71}$ found that $H$. pylori infection upregulated the expression of TRPV1 and NGF genes in human gastric cell lines. The expression of TRPV1 and NGF genes in gastric mucosa of successful $H$. pylori eradication patients was significantly reduced compared with patients without $H$. pylori eradication after 1 year follow-up, and the decreased expression of TRPV 1 and NGF genes was correlated with the improvement of symptoms of FD patients. ${ }^{71}$ It was also demonstrated that NGF, TRPV1, SP, and CGRP were involved in the development of visceral hypersensitivity in patients with IBS. For example, the high level of NGF in the mucosa of rectum and sigmoid was related to the increase of sensory nerve fibers expressing TRPV1 and CGRP in patients with IBS-FD. ${ }^{39}$ The expression of microRNA-199 was significantly reduced in patients with IBS-D and was related to visceral pain since the upregulation of microRNA-199 decreased visceral pain via inhibition of TRPV1 signals. ${ }^{72}$ However, no study have attempted to confirm the relationship between the above mechanism and $H$. pylori infection in patients with IBS.

Eradication of $H$. pylori improved the symptoms of some patients with FD. Therefore, the Rome IV Committee recommended eradication treatment for $H$. pylori-positive patients with FD. ${ }^{73}$ A number of studies have explored the efficacy of $H$. pylori eradication in patients with IBS but no consistent conclusions have been reached. ${ }^{74,75}$ The positive rate of $H$. pylori in patients with IBS-FD and whether $H$. pylori eradication benefits patients with IBS-FD have not been reported. 


\section{The Role of Non-infectious Inflammation in the Pathogenesis of Irritable Bowel Syn- drome, Functional Dyspepsia, and Overlap Syndrome of Irritable Bowel Syndrome and Functional Dyspepsia}

\section{Small Intestinal Bacterial Overgrowth}

In IBS and FD patients without gastrointestinal infection history, the continuous low-grade inflammation of the intestinal mucosa may also be related to small intestinal bacterial overgrowth (SIBO), since intestinal flora makes significant difference in mucosal and systemic immune response. In recent years, SIBO is considered to be significantly associated with functional bowel disease, especially IBS. $^{76} \mathrm{~A}$ recent meta-analysis showed that the incidence of SIBO in IBS patients is $36.7 \%$ (95\% CI, 24.2-44.6) and it was closely associated with intestinal inflammation. ${ }^{77,78}$ There are few studies focusing on the relationship between SIBO and FD. In a small sample Brazilian study, SIBO was found in $56.5 \%$ (13/23) of patients with FD but not observed in healthy controls $(P=0.005) .{ }^{79}$ Japanese scholars tested SIBO in 38 patients with refractory functional gastrointestinal diseases (11 FD, 10 IBS, and 17 IBS-FD) and 2 patients were positive (1 FD and 1 IBS-FD). The symptoms of 2 patients with positive SIBO were significantly improved and their breath hydrogen levels decreased to normal following levofloxacin administration for 7 days, suggesting that the occurrence of FD symptoms may be related to $\mathrm{SIBO}^{80}$

SIBO occurs because bacterium in the distal intestine move into the small intestine for various reasons, which may cause malnutrition, abnormal intestinal motility, diarrhea, abdominal distention, and other symptoms. The overgrowth of harmful bacterium produces a variety of toxic substances including ammonia, Dlactate, endogenous bacterial peptidoglycan, etc. These substances as well as bacterium themselves stimulate intestinal immune cells to produce pro-inflammatory cytokines resulting in sustained mild intestinal inflammation and immune activation, impaired intestinal mucosal barrier function, and increased intestinal sensitivity. It was confirmed that the increase of IL- $1 \alpha$ and IL-1 $\beta$ in the upper gastrointestinal mucosa of patients with IBS was associated with SIBO ${ }^{81}$ Bacterial translocation was associated with enhanced local immune response in the small intestinal mucosa including the increase of plasma cells secreting $\operatorname{IgA}$ and $\operatorname{IgM}$ in the lamina propria. ${ }^{82}$ Clinical research demonstrated that rifaximin effectively relieved the abdominal distention symptoms of IBS or FD patients and the incidence of adverse reactions was low in long-term followup. ${ }^{83,84}$ Rifaximin is a type of oral broad-spectrum antibiotic acting on the local gastrointestinal tract. According to the study on a stressinduced rat model, rifaximin reduced the total load of intestinal flora, increased the relative abundance of lactobacilli, regulated the imbalance between the levels of pro-inflammatory factors and anti-inflammatory factors caused by stress (that is, downregulated the levels of pro-inflammatory factors such as IL-17, IL-6, and TNF- $\alpha$ and upregulated the level of anti-inflammatory factor IL10) so as to inhibit stress-induced chronic inflammation of mucosa, reduce intestinal permeability, and improve visceral hypersensitivity. ${ }^{85}$ At present, lack of large-scale studies, research focuses on the incidence of SIBO in patients with IBS-FD, the correlation between SIBO and overlapping symptoms, and the benefits from rifaximin treatment in patients with IBS-FD.

\section{Food Allergy}

Dietary factors play a more and more vital role in the etiology and pathophysiology of IBS and FD. In addition to their direct effects on sensitive mucosal receptors, food components also participate in the pathogenesis of IBS and FD by inducing mucosal immune response. In IBS patients with food allergy, acute eosinophil degranulation in duodenum and permeability of intestinal mucosa increased, and these patients had a 4-fold increase in prevalence of atopic disorders compared with controls. ${ }^{86}$ In the study of FritscherRavens et $\mathrm{al}^{87}$ diluted food antigens were administered directly to the duodenal mucosa, which immediately caused duodenal mucosal breaks, increased intervillous spaces, and increased intraepithelial lymphocytes in IBS patients with a suspected food intolerance to candidate food antigens. Duodenal eosinophilia of patients with FD was associated with history of allergy (including food allergy) and early satiety. ${ }^{88}$ In addition, $\alpha$-amylase/trypsin inhibitors in wheat act as strong activators of innate immune responses in monocytes, macrophages, and dendritic cells induced gastrointestinal inflammation by activating TLR-4. ${ }^{89}$

In type I anaphylaxis, the combination of $\mathrm{IgE}$ antibody and food allergens induces degranulation of MCs and the recruitment of eosinophils, basophils, and T-lymphocytes in the intestinal mucosa. These cells proliferate and secrete T helper 2 cytokines including IL-4, IL-5, and IL-13. Tryptase released by MCs activates eosinophils through PAR-2 receptor expressed on eosinophils. Activated eosinophils degranulate and release major basic proteins, a type of endogenous allosteric inhibitor of agonist binding to the M2 muscarinic receptor, resulting in the enhancement of smooth muscle contraction. ${ }^{90}$ Early studies have found that IBS patients produced 
higher IgG4 titers for certain dietary antigens (such as wheat, beef, pork, and lamb) compared to controls, and a food elimination diet based on serum IgG/IgG4 antibodies was able to improve overall symptoms in IBS patients. ${ }^{91}$ Similarly, FD patients had significantly higher titers of $\operatorname{IgG}$ antibody to egg and soybean than controls. ${ }^{92}$ It is suggested that IBS or FD symptoms caused by dietary antigens may be involved in the IgG mediated MCs sensitization.

\section{Psychological or Mental Stress}

Studies on animals and humans both have demonstrated that stress was closely correlated with the activation of inflammation and immune response, and its possible mechanism includes the efferent cholinergic pathway of the vagus and the pro-inflammatory and anti-inflammatory effects of central and peripheral CRF signaling pathways. ${ }^{93}$ Compared with control group rats, the number of CD4+ T lymphocytes and the expression of occludin and ZO-1 in the duodenum mucosa of the stressed rats decreased significantly. ${ }^{94}$ The results showed that acute stress could lead to abnormal immune function of the duodenal mucosa, damage of mucosal barrier, and increased permeability of intestine. In addition, the secretion of $\mathrm{CRF}$ by the hypothalamus increased under stress, which promoted the degranulation of MCs in ileum of pigs and the release of TNF- $\alpha$ and tryptase, leading to the increase of intestinal permeability. ${ }^{95}$ The activation of HPA axis and the increase of intestinal mucosal permeability induced by public speech and intravenous injection of CRF could be inhibited by the MC stabilizer sodium cromoglycate, indicating that MCs participated in the inflammatory response mediated by $\mathrm{CRF}^{96}$ Moreover, the activation of HPA axis was associated with the increase of IL-6 in peripheral blood. ${ }^{97}$ Other studies confirmed the relationship between inflammation and mental disorders showed that patients with anxiety and depression had immune dysfunction manifesting as the increase of serum $\mathrm{C}$ reactive protein, IL-6, IL-5, IL-13, TNF- $\alpha$, and other inflammatory mediators. ${ }^{98,99}$ Patients with IBS-FD had more serious psychological problems than those without overlapping symptoms. ${ }^{100}$ Multivariate logistic regression analysis showed that anxiety was an independent factor affecting the overlap of IBS and FD symptoms. ${ }^{101}$ Antidepressants are used in practice for FGIDs patients with epigastric or lower abdominal pain. Current evidence for the use of antidepressants is much stronger in IBS than in FD.

In summary, SIBO, food allergy and mental or psychological stress all participate in the local or systemic chronic inflammatory response and immune dysfunction of IBS/FD as stimulants. The changes of MCs, eosinophils and lymphocytes, and the increase of pro-inflammatory cytokines released by them lead to the increase of intestinal permeability and the disorder of the brain-gut axis, resulting in gastrointestinal symptoms through mechanisms similar to that of PI-IBS/PI-FD.

\section{The Guiding Significance of Inflammatory Mechanism in the Treatment of Patients With Overlap Syndrome of Irritable Bowel Syndrome and Functional Dyspepsia}

Therapies such as food elimination diet, $H$. pylori eradication, antibiotics and antidepressants/anti-anxiety agents (currently called neuromodulators) ${ }^{102}$ can remove the initiating factors of inflammatory response and become potential therapeutic options for IBS-FD patients. However, there is no case-control study to confirm the efficacy of the above treatments in patients with IBS-FD. Other treatments that may be effective for patients with IBS-FD include drugs that inhibit the intermediate links of inflammation besides rifaximin as mentioned above.

Mesalazine plays an anti-inflammatory role through affecting various mediators and signaling pathways that mediate leukocyte chemotaxis and epithelial defense function (such as free radicalscavenging, promoting proliferation of intestinal epithelial cells, and inhibiting their apoptosis). In recent years, the therapeutic effect of mesalazine on intestinal inflammation of IBS patients has been widely reported. It was confirmed that mesalazine reduced the number of MCs in the colonic mucosa of IBS patients and downregulated the function of $\mathrm{MCs}{ }^{103} \mathrm{~A}$ randomized controlled study of Barbara et $\mathrm{al}^{104}$ showed that mesalazine treatment was not superior to placebo at the primary end point of the study (significant improvement of abdominal pain/discomfort), but some IBS patients (11.6\%) showed sustained response (relief of abdominal pain and improvement of overall symptoms) to the treatment. As altered mucosal immune activity is a pivotal pathogenic factor in PI-IBS, Andresen et $\mathrm{al}^{105}$ observed that mesalazine reduced the risk of PI-IBS after infection with Shiga-like toxin-producing E. coli O104:H4 through its modulatory action on mucosal immunity. Since mesalazine mainly acts on the lower gastrointestinal tract to exert its antiinflammatory effect and considering the potential side effects of its long-term treatment, we can speculate that mesalazine benefits little to patients with FD or IBS-FD, but there are no relevant studies.

MCs are the main inflammatory cells involved in the inflammatory response of IBS and FD. Pretreatment with disodium cromoglycate, a MC stabilizer, could prevent the increased intestinal permeability due to acute stress and CRF injection. ${ }^{96}$ Oral disodium cromoglycate treatment could also significantly improve 
gastrointestinal symptoms of patients with IBS by reducing activation of MCs. ${ }^{106}$ Meta-analysis showed that the improvement rate of FD symptoms after being treated by $5-\mathrm{HT}_{4}$ receptor partial agonist (tegaserod) was significantly higher than placebo. ${ }^{107}$ Tegaserod was also significantly better than placebo in relieving IBS symptoms such as abdominal distention and constipation. ${ }^{108} 5-\mathrm{HT}_{3}$ receptor antagonists (alosetron and cilansetron) were more effective than the comparators in achieving global improvement in IBS symptoms and relief of abdominal pain and discomfort. ${ }^{109}$ Through inhibiting the synthesis of 5-HT, tryptophan hydroxylase inhibitor could down-regulate the release of 5-HT in inflammatory response, which is beneficial to the improvement of symptoms in patients with IBS-D. ${ }^{110}$ In the future, more studies will be needed to explore the efficacy of these drugs in patients with IBS-FD.

\section{Summary and Outlook}

The subtype analyses regarding IBS-FD showed that IBS with constipation was the most prevalent subtype in the postprandial distress syndrome-IBS overlap group, while IBS with diarrhea was the most frequently reported type in the EPS-IBS group, ${ }^{111}$ which suggest that visceral hypersensitivity or dyskinesia of the whole gastrointestinal tract and abnormal perception and processing to pain in the central nervous system may be the pathophysiological characteristics of IBS-FD. As mentioned earlier, comorbidity with psychological or mental illness also increases the risk of overlap syndrome.

Table. The Known and Possible Mechanism of Inflammation in Patients With Overlap Syndrome of Irritable Bowel Syndrome and Functional Dyspepsia

\begin{tabular}{|c|c|c|c|c|}
\hline $\begin{array}{l}\text { Pathogenic } \\
\text { factors }\end{array}$ & Disease & Pathophysiological changes & Pathophysiological outcomes & Related symptoms \\
\hline \multirow{10}{*}{$\begin{array}{l}\text { Gastrointestinal } \\
\text { infection }\end{array}$} & \multirow[t]{6}{*}{ IBS } & \multirow{2}{*}{$\begin{array}{l}\text { Intestinal MCs, } T \text { lymphocytes } \uparrow^{12,13} \text { and } \\
\text { inflammatory cytokines } \uparrow^{19,20}\end{array}$} & Increased intestinal permeability & \multirow{6}{*}{$\begin{array}{l}\text { Diarrhea }^{33} \\
\text { Abdominal pain }\end{array}$} \\
\hline & & & Visceral hypersensitivity & \\
\hline & & $\begin{array}{l}\text { Downregulation and redistribution of } \\
\mathrm{ZO}-1 \text { and occludin }\end{array}$ & Colon transit and defecation $\uparrow^{61,62}$ & \\
\hline & & TRPV1, SP, and CGRP positive nerve fibers $\uparrow^{38,39}$ & & \\
\hline & & $\begin{array}{l}\text { Increased excitability of DRG and } \\
\text { submucous plexus neurons }{ }^{54,55}\end{array}$ & & \\
\hline & & $\begin{array}{l}\text { Persistent activation of CRF neurons, } \\
\text { CRF binding to } \mathrm{CRF}_{1} \text { receptor } \\
61,62\end{array}$ & & \\
\hline & \multirow[t]{4}{*}{ FD } & Gastric and duodenal MCs, eosinophils $\uparrow^{14-16}$ & Damage of duodenal mucosal barrier & \\
\hline & & Abdominal expression of $\mathrm{ZO}-1$ and occludin ${ }^{36}$ & Visceral hypersensitivity & \multirow{3}{*}{ Early satiety $^{18}$} \\
\hline & & SP, CGRP positive nerve fibers $\uparrow^{41,42}$ & Gastric contraction and & \\
\hline & & $\mathrm{CRF}$ binding to $\mathrm{CRF}_{2}$ receptor ${ }^{61,62}$ & gastric emptying $\downarrow^{61,62}$ & \\
\hline \multirow[t]{2}{*}{ SIBO } & IBS & IL- $1 \alpha$ and IL- $1 \beta \uparrow^{81}$ & $\begin{array}{l}\text { Mild inflammation and } \\
\text { immune activation of } \\
\text { intestinal mucosa }\end{array}$ & $\begin{array}{l}\text { Abdominal distention } \\
\text { relieved by rifaximin }^{83}\end{array}$ \\
\hline & FD & NA & NA & $\begin{array}{l}\text { Abdominal distention } \\
\text { relieved by rifaximin }^{84}\end{array}$ \\
\hline \multirow[t]{4}{*}{ Food allergy } & \multirow[t]{2}{*}{ IBS } & $\begin{array}{l}\text { Increased intervillous spaces and } \\
\text { intraepithelial lymphocytes in intestinal mucosa }\end{array}$ & \multirow[t]{2}{*}{ Increased intestinal permeability } & \\
\hline & & Titers of IgG antibody to specific food antigen $\uparrow^{91}$ & & \\
\hline & \multirow[t]{2}{*}{ FD } & Duodenal eosinophilia $^{88}$ & NA & \multirow[t]{2}{*}{ Early satiety $^{88}$} \\
\hline & & Titers of IgG antibody to specific food antigen $\uparrow^{92}$ & & \\
\hline \multirow{3}{*}{$\begin{array}{l}\text { Psychological or } \\
\text { mental stress }\end{array}$} & & CD4 + T lymphocytes $\downarrow^{94}$ & \multirow{3}{*}{$\begin{array}{l}\text { Increased intestinal permeability } \\
\text { Visceral hypersensitivity }\end{array}$} & \\
\hline & & $\begin{array}{l}\text { CRF secreted by hypothalamus } \uparrow \text {, degranulation of } \\
\text { MCs and released TNF- } \alpha \text { and tryptase } \uparrow^{95}\end{array}$ & & \\
\hline & & $\begin{array}{l}\text { Expression of occludin and ZO-1 in } \\
\text { duodenal mucosal } \downarrow^{94}\end{array}$ & & \\
\hline
\end{tabular}

SIBO, small intestinal bacterial overgrowth; IBS, irritable bowel syndrome; FD, functional dyspepsia; MC, mast cell; ZO, zonula occludens; TRPV1, transient receptor potential vanilloid type-1; SP, substance P; CGRP, calcitonin gene related peptide; DRG, dorsal root ganglion; CRF, corticotropin releasing factor; NA, not applicable. 
It is worth mentioning that inflammation may be involved in any level of the above pathogenesis. The mechanism of inflammation in IBS-FD can be summarized as various factors such as acute gastrointestinal infection, intestinal flora disorder, food allergy, or stress lead to the damage of intestinal epithelial barrier and antigen presentation, causing the activation of inflammatory cells and release of pro-inflammatory cytokines and chemokines of inflammatory cells. Then, the activation and degranulation of inflammatory cells leads to different degree of gastrointestinal mucosal inflammation and immune response resulting in visceral hypersensitivity, dysfunction of gastrointestinal motility, sensory and secretion. Compared with IBS or FD patients, the systemic or local inflammation of the gastrointestinal tract induced by infection, diet, microbiota alteration, mental or psychological factors may be more obvious in patients with IBS-FD. In addition, the distribution of inflammation is probably more extensive in the digestive tract, which involves the upper and lower digestive tract at the same time or subsequently, resulting in overlapping symptoms.

The known and possible mechanisms of inflammation in patients with IBS-FD is summarized in Table. Although there are few studies regarding IBS-FD, this article specifically reveals many commonalities of inflammation in the mechanism of IBS and FD and provides references for future research direction. In the future, we need more researches focusing on confirming the pathogenesis of IBS-FD, and more high-quality, multi-center, and large-sample studies on efficacy to guide clinicians to optimize the treatment of patients with IBS-FD.

Financial support: This study was supported by The National Natural Science Foundation of China (81870379) and The Integrative Project of Peking Union Medical College Hospital (ZC201903088).

\section{Conflicts of interest: None.}

Author contributions: Congzhen Wang performed the literature search, drafted the article, and revised the article based on the comments of Xiucai Fang; and Xiucai Fang contributed to the study design, revising the article, and final approval to the submission.

\section{References}

1. Kibune Nagasako C, Garcia Montes C, Silva Lorena SL, Mesquita MA. Irritable bowel syndrome subtypes: clinical and psychological features, body mass index and comorbidities. Rev Esp Enferm Dig
2016;108:59-64.

2. Perveen I, Rahman MM, Saha M, Rahman MM, Hasan MQ. Prevalence of irritable bowel syndrome and functional dyspepsia, overlapping symptoms, and associated factors in a general population of Bangladesh. Indian J Gastroenterol 2014;33:265-273.

3. Sun YF, Li YQ, Wang YP. The feature of clinical symptoms and healthrelated quality of life in patients with overlapping functional dyspepsia and irritable bowel syndrome. Chin J Gastroenterol 2006;11:492-494.

4. von Wulffen M, Talley NJ, Hammer J, et al. Overlap of irritable bowel syndrome and functional dyspepsia in the clinical setting: prevalence and risk factors. Dig Dis Sci 2019;64:480-486.

5. Futagami S, Itoh T, Sakamoto C. Systematic review with metaanalysis: post-infectious functional dyspepsia. Aliment Pharmacol Ther 2015;41:177-188.

6. Shariati A, Fallah F, Pormohammad A, et al. The possible role of bacteria, viruses, and parasites in initiation and exacerbation of irritable bowel syndrome. J Cell Physiol 2019;234:8550-8569.

7. Wadhwa A, Al Nahhas MF, Dierkhising RA, et al. High risk of postinfectious irritable bowel syndrome in patients with Clostridium difficile infection. Aliment Pharmacol Ther 2016;44:576-582.

8. Rahman MM, Ghoshal UC, Sultana S, et al. Long-term gastrointestinal consequences are frequent following sporadic acute infectious diarrhea in a tropical country: a prospective cohort study. Am J Gastroenterol 2018;113:1363-1375

9. Mearin F, Pérez-Oliveras M, Perelló A, et al. Dyspepsia and irritable bowel syndrome after a Salmonella gastroenteritis outbreak: one-year follow-up cohort study. Gastroenterology 2005;129:98-104 .

10. Wensaas KA, Hanevik K, Hausken T, et al. Postinfectious and sporadic functional gastrointestinal disorders have different prevalences and rates of overlap: results from a controlled cohort study 3 years after acute giardiasis. Neurogastroenterol Motil 2016;28:1561-1569.

11. Spiller RC. Postinfectious functional dyspepsia and postinfectious irritable bowel syndrome: different symptoms but similar risk factors. Gastroenterology 2010;138:1660-1663.

12. Wang LH, Fang XC, Pan GZ. Bacillary dysentery as a causative factor of irritable bowel syndrome and its pathogenesis. Gut 2004;53:10961101.

13. Spiller RC, Jenkins D, Thornley JP, et al. Increased rectal mucosal enteroendocrine cells, $\mathrm{T}$ lymphocytes, and increased gut permeability following acute Campylobacter enteritis and in post-dysenteric irritable bowel syndrome. Gut 2000;47:804-811.

14. Li X, Chen H, Lu H, et al. The study on the role of inflammatory cells and mediators in postinfectious functional dyspepsia. Scand J Gastroenterol 2010;45:573-581.

15. Kindt S, Tertychnyy A, de Hertogh G, Geboes K, Tack J. Intestinal immune activation in presumed post-infectious functional dyspepsia. Neurogastroenterol Motil 2009;21:832-e56.

16. Futagami S, Shindo T, Kawagoe T, et al. Migration of eosinophils and CCR2-/CD68-double positive cells into the duodenal mucosa of patients with postinfectious functional dyspepsia. Am J Gastroenterol 2010;105:1835-1842.

17. Barbara G, Stanghellini V, De Giorgio R, et al. Activated mast cells in 
proximity to colonic nerves correlate with abdominal pain in irritable bowel syndrome. Gastroenterology 2004;126: 693-702.

18. Walker MM, Aggarwal KR, Shim LS, et al. Duodenal eosinophilia and early satiety in functional dyspepsia: confirmation of a positive association in an Australian cohort. J Gastroenterol Hepatol 2014;29:474-479.

19. Liang HQ, Wang SH, Zhu Y, Wang FS. The imbalance of intestinal mucosa inflammatory cytokine in patients with post-infection irritable bowel syndrome. Chin J Gastroenterol Hepatol 2010;19:1032-1035.

20. Chen J, Zhang Y, Deng Z. Imbalanced shift of cytokine expression between $\mathrm{T}$ helper 1 and $\mathrm{T}$ helper 2 (Th1/Th2) in intestinal mucosa of patients with post-infectious irritable bowel syndrome. BMC Gastroenterol 2012;12:91.

21. Liang H, Wang S, Li Y, Wang F. Analysis on expression imbalance of peripheral blood inflammatory cytokines in patients with irritable bowel syndrome. Chin J Gastroenterol 2008;13:111-113.

22. Schmulson M, Pulido-London D, Rodriguez O, et al. Lower serum IL-10 is an independent predictor of IBS among volunteers in Mexico. Am J Gastroenterol 2012;107:747-753.

23. Alonso C, Vicario M, Pigrau M, Lobo B, Santos J. Intestinal barrier function and the brain-gut axis. Adv Exp Med Biol 2014;817:73-113.

24. Bhattarai Y, Muniz Pedrogo DA, Kashyap PC. Irritable bowel syndrome: a gut microbiota-related disorder? Am J Physiol Gastrointest Liver Physiol 2017;312:G52-G62.

25. Faure M, Moënnoz D, Montigon F, Mettraux C, Breuillé D, Ballèvre O. Dietary threonine restriction specifically reduces intestinal mucin synthesis in rats. J Nutr 2005;135:486-491.

26. Wrzosek L, Miquel S, Noordine ML, et al. Bacteroides thetaiotaomicron and Faecalibacterium prausnitzii influence the production of mucus glycans and the development of goblet cells in the colonic epithelium of a gnotobiotic model rodent. BMC Biol 2013;11:61.

27. Jalanka-Tuovinen J, Salojärvi J, Salonen A, et al. Faecal microbiota composition and host-microbe cross-talk following gastroenteritis and in postinfectious irritable bowel syndrome. Gut 2014;63:1737-1745.

28. Pittayanon R, Lau JT, Yuan Y, et al. Gut microbiota in patients with irritable bowel syndrome-a systematic review. Gastroenterology 2019;157:97-108.

29. Sundin J, Rangel I, Fuentes S, et al. Altered faecal and mucosal microbial composition in post-infectious irritable bowel syndrome patients correlates with mucosal lymphocyte phenotypes and psychological distress. Aliment Pharmacol Ther 2015;41:342-351.

30. Zhong L, Shanahan ER, Raj A, et al. Dyspepsia and the microbiome: time to focus on the small intestine. Gut 2017;66:1168-1169.

31. Anderson JM. Molecular structure of tight junctions and their role in epithelial transport. News Physiol Sci 2001;16:126-130.

32. Tsukita S, Katsuno T, Yamazaki Y, Umeda K, Tamura A, Tsukita S. Roles of ZO-1 and ZO-2 in establishment of the belt-like adherens and tight junctions with paracellular permselective barrier function. Ann N Y Acad Sci 2009;1165:44-52.

33. Martinez C, Vicario M, Ramos L, et al. The jejunum of diarrheapredominant irritable bowel syndrome shows molecular alterations in the tight junction signaling pathway that are associated with mucosal pathobiology and clinical manifestations. Am J Gastroenterol 2012;107:736-
746.

34. Chin AC, Vergnolle N, MacNaughton WK, Wallace JL, Hollenberg $\mathrm{MD}$, Buret AG. Proteinase-activated receptor 1 activation induces epithelial apoptosis and increases intestinal permeability. Proc Natl Acad Sci USA 2003;100:11104-11109.

35. Farré R, Vicario M. Abnormal barrier function in gastrointestinal disorders. Handb Exp Pharmacol 2017;239:193-217.

36. Vanheel H, Vicario M, Vanuytsel T, et al. Impaired duodenal mucosal integrity and low-grade inflammation in functional dyspepsia. Gut 2014;63:262-271.

37. Wang LH, Fang XC, Pan GZ. Relationship between mast cells and nerve fibers in the intestinal mucosa in patients with irritable bowel syndrome. Chin J Dig 2003;23:332-335.

38. van den Wijngaard RM, Klooker TK, Welting O, et al. Essential role for TRPV1 in stress-induced (mast cell-dependent) colonic hypersensitivity in maternally separated rats. Neurogastroenterol Motil 2009;21:1107-e94.

39. Xu XJ, Zhang YL, Liu L, Pan L, Yao SK. Increased expression of nerve growth factor correlates with visceral hypersensitivity and impaired gut barrier function in diarrhoea-predominant irritable bowel syndrome: a preliminary explorative study. Aliment Pharmacol Ther 2017;45:100114.

40. Balestra B, Vicini R, Cremon C, et al. Colonic mucosal mediators from patients with irritable bowel syndrome excite enteric cholinergic motor neurons. Neurogastroenterol Motil 2012;24:1118-e570.

41. Stanzel RD, Lourenssen S, Blennerhassett MG. Inflammation causes expression of NGF in epithelial cells of the rat colon. Exp Neurol 2008;211:203-213.

42. Demir IE, Schäfer KH, Tieftrunk E, Friess H, Ceyhan GO. Neural plasticity in the gastrointestinal tract: chronic inflammation, neurotrophic signals, and hypersensitivity. Acta Neuropathol 2013;125:491-509.

43. Lee MJ, Jung HK, Lee KE, Mun YC, Park S. Degranulated eosinophils contain more fine nerve fibers in the duodenal mucosa of patients with functional dyspepsia. J Neurogastroenterol Motil 2019;25:212221.

44. Cirillo C, Bessissow T, Desmet AS, Vanheel H, Tack J, Vanden Berghe P. Evidence for neuronal and structural changes in submucous ganglia of patients with functional dyspepsia. Am J Gastroenterol 2015;110:12051215.

45. Stasi C, Bellini M, Bassotti G, Blandizzi C, Milani S. Serotonin receptors and their role in the pathophysiology and therapy of irritable bowel syndrome. Tech Coloproctol 2014;18:613-621.

46. Zhang LY, Dong X, Liu ZL, et al. Luminal serotonin time-dependently modulates vagal afferent driven antinociception in response to colorectal distention in rats. Neurogastroenterol Motil 2011;23:62-69, e6.

47. Spiller RC, Jenkins D, Thornley JP, et al. Increased rectal mucosal enteroendocrine cells, $\mathrm{T}$ lymphocytes, and increased gut permeability following acute Campylobacter enteritis and in post-dysenteric irritable bowel syndrome. Gut 2000;47:804-811.

48. Dunlop SP, Jenkins D, Neal KR, Spiller RC. Relative importance of enterochromaffin cell hyperplasia, anxiety, and depression in postinfectious IBS. Gastroenterology 2003;125:1651-1659. 
49. Foley KF, Pantano C, Ciolino A, Mawe GM. IFN-gamma and TNFalpha decrease serotonin transporter function and expression in $\mathrm{Caco} 2$ cells. Am J Physiol Gastrointest Liver Physiol 2007;292:G779-G784.

50. Gaman A, Kuo B. Neuromodulatory processes of the brain-gut axis. Neuromodulation 2008;11:249-259.

51. Jones MP, Dilley JB, Drossman D, Crowell MD. Brain-gut connections in functional GI disorders: anatomic and physiologic relationships. Neurogastroenterol Motil 2006;18:91-103.

52. Johnson AC, Myers B, Lazovic J, Towner R, Greenwood-Van Meerveld B. Brain activation in response to visceral stimulation in rats with amygdala implants of corticosterone: an FMRI study. PLoS One 2010;5:e8573.

53. Furuta S, Shimizu T, Narita M, et al. Subdiaphragmatic vagotomy promotes nociceptive sensitivity of deep tissue in rats. Neuroscience 2009;164:1252-1262.

54. Barbara G, Wang B, Stanghellini V, et al. Mast cell-dependent excitation of visceral-nociceptive sensory neurons in irritable bowel syndrome. Gastroenterology 2007;132:26-37.

55. Buhner S, Li Q, Berger T, et al. Submucous rather than myenteric neurons are activated by mucosal biopsy supernatants from irritable bowel syndrome patients. Neurogastroenterol Motil 2012;24:1134-e572.

56. Furness JB, Rivera LR, Cho HJ, Bravo DM, Callaghan B. The gut as a sensory organ. Nat Rev Gastroenterol Hepatol 2013;10:729-740.

57. Gershon MD, Tack J. The serotonin signaling system: from basic understanding to drug development for functional GI disorders. Gastroenterology 2007;132:397-414.

58. Martínez V, Wang L, Rivier J, Grigoriadis D, Taché Y. Central CRF, urocortins and stress increase colonic transit via $\mathrm{CRF} 1$ receptors while activation of CRF2 receptors delays gastric transit in mice. J Physiol 2004;556(pt 1):221-234.

59. Fichna J, Storr MA. Brain-gut interactions in IBS. Front Pharmacol 2012;3:127.

60. Overman EL, Rivier JE, Moeser AJ. CRF induces intestinal epithelial barrier injury via the release of mast cell proteases and TNF- $\alpha$. PLoS One 2012; 7:e39935.

61. Hussain Z, Kim HW, Huh CW, Lee YJ, Park H. The effect of peripheral CRF peptide and water avoidance stress on colonic and gastric transit in guinea pigs. Yonsei Med J 2017;58:872-877.

62. Hussain Z, Jung DH, Lee YJ, Park H. The effect of trimebutine on the overlap syndrome model of guinea pigs. J Neurogastroenterol Motil 2018;24:669-675.

63. Ng QX, Foo NX, Loke W, et al. Is there an association between Helicobacter pylori infection and irritable bowel syndrome? A meta-analysis. World J Gastroenterol 2019;25:5702-5710.

64. Yakoob J, Abbas Z, Naz S, Islam M, Jafri W. Virulence markers of Helicobacter pylori in patients with diarrhoea-dominant irritable bowel syndrome. Br J Biomed Sci 2012;69:6-10.

65. Supajatura V, Ushio H, Wada A, et al. Cutting edge: VacA, a vacuolating cytotoxin of Helicobacter pylori, directly activates mast cells for migration and production of proinflammatory cytokines. J Immunol 2002;168:2603-2607.

66. Montemurro P, Nishioka H, Dundon WG, et al. The neutrophil-acti- vating protein (HP-NAP) of Helicobacter pylori is a potent stimulant of mast cells. Eur J Immunol 2002;32:671-676.

67. Capannolo A, Necozione S, Gabrieli D, et al. Duodenal lymphocytosis in functional dyspepsia. Arab J Gastroenterol 2019;20:91-94.

68. Mirbagheri SA, Khajavirad N, Rakhshani N, Ostovaneh MR, Hoseini SM, Hoseini V. Impact of Helicobacter pylori infection and microscopic duodenal histopathological changes on clinical symptoms of patients with functional dyspepsia. Dig Dis Sci 2012;57:967-972.

69. Ford AC, Forman D, Bailey AG, Axon AT, Moayyedi P. Irritable bowel syndrome: a 10-year natural history of symptoms and factors that influence consultation behavior. Am J Gastroenterol 2008;103:1229-1239.

70. Mönnikes H, van der Voort IR, Wollenberg B, et al. Gastric perception thresholds are low and sensory neuropeptide levels high in Helicobacter pylori-positive functional dyspepsia. Digestion 2005;71:111-123.

71. Choi YJ, Kim N, Kim J, Lee DH, Park JH, Jung HC. Upregulation of vanilloid receptor-1 in functional dyspepsia with or without Helicobacter pylori infection. Medicine (Baltimore) 2016;95:e3410.

72. Zhou Q, Yang L, Larson S, et al. Decreased miR-199 augments visceral pain in patients with IBS through translational upregulation of TRPV1. Gut 2016;65:797-805.

73. Du LJ, Chen BR, Kim JJ, Kim S, Shen JH, Dai N. Helicobacter pylori eradication therapy for functional dyspepsia: systematic review and metaanalysis. World J Gastroenterol 2016;22: 3486-3495.

74. Xiong F, Xiong M, Ma Z, Huang S, Li A, Liu S. Lack of association found between Helicobacter pylori infection and diarrhea-predominant irritable bowel syndrome: a multicenter retrospective study. Gastroenterol Res Pract 2016;2016:3059201.

75. Li XQ, Wu YS, Pan LJ, Tao ZQ. The relationship between Helicobacter pylori infection and irritable bowel syndrome. Chin J Gastroenterol Hepatol 2012;21:706-707.

76. Moraru IG, Moraru AG, Andrei M, et al. Small intestinal bacterial overgrowth is associated to symptoms in irritable bowel syndrome. Evidence from a multicentre study in Romania. Rom J Intern Med 2014;52:143-150.

77. Ghoshal UC, Nehra A, Mathur A, Rai S. A meta-analysis on small intestinal bacterial overgrowth in patients with different subtypes of irritable bowel syndrome. J Gastroenterol Hepatol 2020;35:922-931.

78. David L, Babin A, Picos A, Dumitrascu DL. Small intestinal bacterial overgrowth is associated with intestinal inflammation in the irritable bowel syndrome. Clujul Med 2014;87:163-165.

79. Costa MB, Azeredo IL Jr, Marciano RD, Caldeira LM, Bafutto M. Evaluation of small intestine bacterial overgrowth in patients with functional dyspepsia through $\mathrm{H} 2$ breath test. Arq Gastroenterol 2012;49:279-283.

80. Shimura S, Ishimura N, Mikami H, et al. Small intestinal bacterial overgrowth in patients with refractory functional gastrointestinal disorders. J Neurogastroenterol Motil 2016;22:60-68.

81. Srivastava D, Ghoshal U, Mittal RD, Ghoshal UC. Associations between IL-1RA polymorphisms and small intestinal bacterial overgrowth among patients with irritable bowel syndrome from India. Neurogastroenterol Motil 2014;26:1408-1416.

82. Woodcock NP, Robertson J, Morgan DR, Gregg KL, Mitchell CJ, 
MacFie J. Bacterial translocation and immunohistochemical measurement of gut immune function. J Clin Pathol 2001;54:619-623.

83. Rivkin A, Rybalov S. Update on the management of diarrhea-predominant irritable bowel syndrome: focus on rifaximin and eluxadoline. Pharmacotherapy 2016;36:300-316.

84. Tan VP, Liu KS, Lam FY, Hung IF, Yuen MF, Leung WK. Randomised clinical trial: rifaximin versus placebo for the treatment of functional dyspepsia. Aliment Pharmacol Ther 2017;45:767-776.

85. Xu D, Gao J, Gillilland M 3rd, et al. Rifaximin alters intestinal bacteria and prevents stress-induced gut inflammation and visceral hyperalgesia in rats. Gastroenterology 2014;146:484-496, e4.

86. Fritscher-Ravens A, Pflaum T, Mösinger M, et al. Many patients with irritable bowel syndrome have a typical food allergies not associated with immunoglobulin E. Gastroenterology 2019;157:109-118, e5.

87. Fritscher-Ravens A, Schuppan D, Ellrichmann M, et al. Confocal endomicroscopy shows food-associated changes in the intestinal mucosa of patients with irritable bowel syndrome. Gastroenterology 2014;147:1012-1020, e4.

88. Walker MM, Salehian SS, Murray CE, et al. Implications of eosinophilia in the normal duodenal biopsy-an association with allergy and functional dyspepsia. Aliment Pharmacol Ther 2010;31:1229-1236.

89. Junker Y, Zeissig S, Kim SJ, et al. Wheat amylase trypsin inhibitors drive intestinal inflammation via activation of toll-like receptor 4. J Exp Med 2012;209:2395-2408.

90. Jacoby DB, Gleich GJ, Fryer AD. Human eosinophil major basic protein is an endogenous allosteric antagonist at the inhibitory muscarinic M2 receptor. J Clin Invest 1993;91:1314-1318.

91. Cuomo R, Andreozzi P, Zito FP, Passananti V, De Carlo G, Sarnelli G. Irritable bowel syndrome and food interaction. World J Gastroenterol 2014;20:8837-8845.

92. Zuo XL, Li YQ, Li WJ, et al. Alterations of food antigen-specific serum immunoglobulins $\mathrm{G}$ and $\mathrm{E}$ antibodies in patients with irritable bowel syndrome and functional dyspepsia. Clin Exp Allergy 2007;37:823-830.

93. O'Malley D, Quigley EM, Dinan TG, Cryan JF. Do interactions between stress and immune responses lead to symptom exacerbations in irritable bowel syndrome? Brain Behav Immun 2011;25:1333-1341.

94. Lee HS, Kim DK, Kim YB, Lee KJ. Effect of acute stress on immune cell counts and the expression of tight junction proteins in the duodenal mucosa of rats. Gut Liver 2013;7:190-196.

95. Overman EL, Rivier JE, Moeser AJ. CRF induces intestinal epithelial barrier injury via the release of mast cell proteases and TNF- $\alpha$. PLoS One 2012;7:e39935.

96. Vanuytsel T, van Wanrooy S, Vanheel H. Psychological stress and corticotropin-releasing hormone increase intestinal permeability in humans by a mast cell-dependent mechanism. Gut 2014;63:1293-1299.

97. Dinan TG, Quigley EM, Ahmed SM, et al. Hypothalamic-pituitarygut axis dysregulation in irritable bowel syndrome: plasma cytokines as a potential biomarker? Gastroenterology 2006;130:304-311.

98. Blume J, Douglas SD, Evans DL. Immune suppression and immune activation in depression. Brain Behav Immun 2011;25:221-229.

99. Kindt S, Van Oudenhove L, Broekaert D, et al. Immune dysfunction in patients with functional gastrointestinal disorders. Neurogastroenterol Motil 2009;21:389-398.

100. Piacentino D, Cantarini R, Alfonsi M, et al. Psychopathological features of irritable bowel syndrome patients with and without functional dyspepsia: a cross sectional study. BMC Gastroenterol 2011;11:94.

101. Lee SY, Lee KJ, Kim SJ, Cho SW. Prevalence and risk factors for overlaps between gastroesophageal reflux disease, dyspepsia, and irritable bowel syndrome: a population-based study. Digestion 2009;79:196-201.

102. Drossman DA, Tack J, Ford AC, Szigethy E, Törnblom H, Van Oudenhove L. Neuromodulators for functional gastrointestinal disorders (disorders of gut-brain interaction): a rome foundation working team report. Gastroenterology 2018;154:1140-1171, e1.

103. Corinaldesi R, Stanghellini V, Cremon C, et al. Effect of mesalazine on mucosal immune biomarkers in irritable bowel syndrome: a randomized controlled proof-of-concept study. Aliment Pharmacol Ther 2009;30:245-252.

104. Barbara G, Cremon C, Annese V, et al. Randomised controlled trial of mesalazine in IBS. Gut 2016;65:82-90.

105. Andresen V, Löwe B, Broicher W, et al. Post-infectious irritable bowel syndrome (PI-IBS) after infection with Shiga-like toxin-producing Escherichia coli (STEC) O104:H4: a cohort study with prospective follow-up. United European Gastroenterol J 2016;4:121-131.

106. Lobo B, Ramos L, Martínez C, et al. Downregulation of mucosal mast cell activation and immune response in diarrhoea-irritable bowel syndrome by oral disodium cromoglycate: a pilot study. United European Gastroenterol J 2017;5:887-897.

107. Jin M, Mo Y, Ye K, Chen M, Liu Y, He C. Efficacy of serotonin receptor agonists in the treatment of functional dyspepsia: a meta-analysis. Arch Med Sci 2019;15:23-32.

108. Madia VN, Messore A, Saccoliti F, et al. Tegaserod for the treatment of irritable bowel syndrome. Antiinflamm Antiallergy Agents Med Chem 2020;19:342-369.

109. Andresen V, Montori VM, Keller J, West CP, Layer P, Camilleri M. Effects of 5-hydroxytryptamine (serotonin) type 3 antagonists on symptom relief and constipation in nonconstipated irritable bowel syndrome: a systematic review and meta-analysis of randomized controlled trials. Clin Gastroenterol Hepatol 2008;6:545-555.

110. Brown PM, Drossman DA, Wood AJ, et al. The tryptophan hydroxylase inhibitor LX1031 shows clinical benefit in patients with nonconstipating irritable bowel syndrome. Gastroenterology 2011;141:507-516.

111. Choi YJ, Kim N, Yoon H, et al. Overlap between irritable bowel syndrome and functional dyspepsia including subtype analyses. J Gastroenterol Hepatol 2017;32:1553-1561. 